\title{
A hybrid instantaneous recovery route design scheme with two different coding aware scenarios*
}

\author{
Pham Vu Phonga), Abu Hena Al Muktadir, and Eiji Oki \\ Department of Communication Engineering and Informatics, \\ The University of Electro-Communications, Tokyo, Japan \\ a)vuphong@uec.ac.jp
}

\begin{abstract}
This letter proposes a hybrid scheme for instantaneous recovery $1+1$ protection route design for all possible source-destination pairs in a network. In the proposed scheme two different coding aware scenarios, namely the TS (traffic splitting) scenario and the 2SD (two sources and a common destination) scenario, are used intelligently according to the largest effective gain first policy. We compare the total costs of all the working and protection paths obtained by the proposed scheme, the TS scenario, and the 2SD scenario. Numerical results observe that the proposed scheme achieves more resource saving than the TS or 2SD scenario in our examined networks.
\end{abstract}

Keywords: $1+1$ protection, instantaneous recovery, traffic splitting, hybrid scheme, coding

Classification: Internet

\section{References}

[1] A. H. A. Muktadir and E. Oki, "Optimum route design in $1+1$ protection with network coding for instantaneous recovery," IEICE Trans. Commun., vol. E97B, no. 1, pp. 87-104, Jan. 2014. DOI:10.1587/transcom.E97.B.87

[2] A. A. Jose, A. H. A. Muktadir, and E. Oki, "Network coding aware instantaneous recovery scheme based on optimal traffic splitting," IEICE Commun. Express, vol. 1, no. 1, pp. 28-32, Jun. 2012. DOI:10.1587/comex.1.28

[3] R. Ahlswede, N. Cai, S. R. Li, and R. W. Yeung, "Network information flow," IEEE Trans. Inf. Theory, vol. 46, no. 4, pp. 1204-1216, Jul. 2000. DOI:10. $1109 / 18.850663$

[4] A. H. A. Muktadir and E. Oki, "A heuristic routing algorithm with erasure correcting code based instantaneous recovery technique," Proc. IEEE ICC 2014 Conf., Sydney, Australia, pp. 1149-1153, Jun. 2014. DOI:10.1109/ICC.2014. 6883475

[5] A. H. A. Muktadir and E. Oki, "A heuristic routing algorithm for network coding aware $1+1$ protection route design for instantaneous recovery," Proc. $15^{\text {th }}$ IEEE HPSR Conf., Vancouver, British Columbia, Canada, pp. 84-89, Jul. 2014. DOI:10.1109/HPSR.2014.6900886

[6] B. C. Chatterjee, N. Sarma, and P. P. Sahu, "Priority based routing and wave- 
length assignment with traffic grooming for optical networks," IEEE/OSA J. Opt. Commun. Netw., vol. 4, no. 6, pp. 480-489, 2012. DOI:10.1364/JOCN.4. 000480

[7] P. V. Phong, A. H. A. Muktadir, and E. Oki, "A hybrid scheme for instantaneous recovery route design with two different coding aware scenarios," Proc. IEEE Int. Conf. on Network Infrastructure and Digital Content (IC-NIDC 2014), Beijing, China, pp. 19-21, Sep. 2014.

[8] Visit http://www.ieice.org/ pn/jpn/jpnm.html, 2014.

\section{Introduction}

$1+1$ protection is a proactive dedicated path protection technique, where data are sent simultaneously along two link disjoint active and protection paths. In $1+1$ protection, $100 \%$ of data can be recovered by switching the working path to the backup path at the destination node when a link failure is detected. $1+1$ protection technique provides instantaneous recovery, in which the recovery action is achieved only at the destination node. However, it requires at least double network resource.

Coding aware $1+1$ protection techniques presented in $[1,2]$ reduces the backup resource requirement for conventional $1+1$ protection. In the $2 \mathrm{SD}$ (two sources and a common destination) scenario, coding can be performed at some intermediate node [1] (known as network coding [3]). In the TS (traffic splitting) scenario [2, 4], coding is performed at the source node with split data.

Theoretically, up to $25 \%$ of resource saving is possible by introducing NC with $1+1$ protection in the 2SD scenario. In Fig. 1(a), let $c_{1}$ be the cost of links $S_{1}-M$ and $S_{2}-M$ and $c_{2}$ be the cost of other links. For conventional $1+1$ protection in this scenario, the total cost is $4 c_{2}+2 c_{1}$. When network coding is employed, link $M-D$ is used only once, and the total required cost is reduced to $3 c_{2}+2 c_{1}$. Thus, $\frac{c_{2}}{4 c_{2}+2 c_{1}} \times 100 \%$ of resource saving is achieved.

Assume that there is an unit traffic demand between the source and destination in Fig. 1(b). This demand is split into $K$ equal parts. Bandwidth demand on each of the used disjoint paths is $\frac{1}{K}$. For conventional $1+1$ protection, the total required bandwidth on two disjoint paths is two. With splitting, this total cost becomes $\frac{K+1}{K}$. Thus, the bandwidth saving, with respect to conventional $1+1$ protection bandwidth requirement, is $\frac{2-\frac{K+1}{K}}{2} \times 100 \%$. If $K$ becomes large, the bandwidth saving approaches to about $50 \%$.

Coding aware instantaneous recovery $1+1$ protection route design for all possible source-destination pairs in the network has been addressed in $[4,5]$. The resource savings or cost reduction results reported in those works (with respect to conventional $1+1$ protection cost) $15 \%$ by using 2 SD [5], and $20 \%$ by using TS [4]. We focus designing routes for all possible source-destination pairs by using TS and 2SD scenarios intelligently. Our motivation is to enhance the resource saving results reported in $[5,4]$ by using multiple protection scenarios according to some policy in our considered route design problem.

One question arises: "How much resource saving is possible if we are allowed to use both TS and 2SD scenarios intelligently for designing coding based protection routes for all possible source-destination pairs?" 


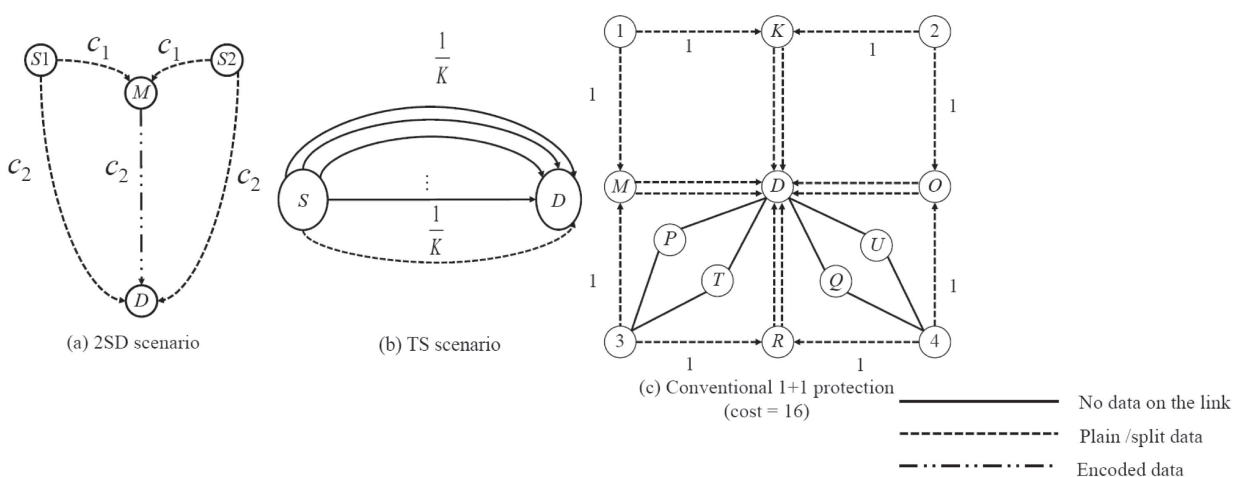

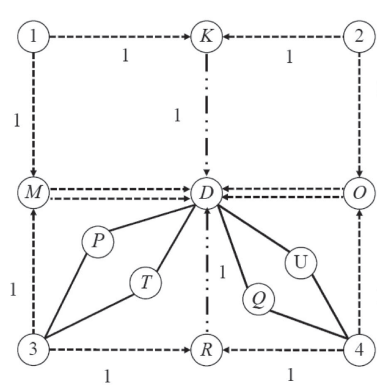

(d) $2 \mathrm{SD}$ scenario based protection $(\cos t=14)$

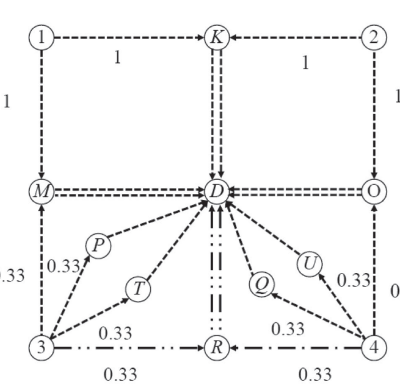

(e) TS scenario based protection $(\cos t=13.28)$

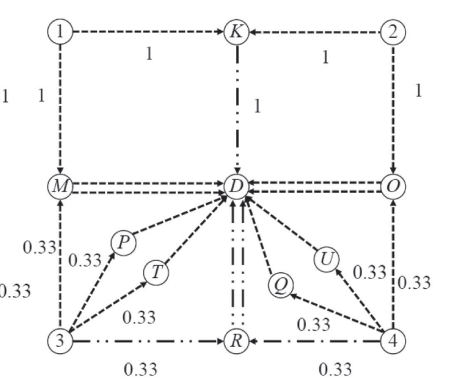

(f) Proposed hybrid scheme $(\operatorname{cost}=12.28)$

Fig. 1. Illustration of various protection scenarios.

To answer this question, this letter proposes a hybrid scheme for instantaneous recovery $1+1$ protection route design. The proposed scheme uses both TS and 2SD intelligently according to the largest effective gain first policy. Numerical results observe that the proposed scheme achieves $1.6-2.1 \%$ more resource saving than either TS or 2SD in our examined networks.

\section{Combining TS and 2SD scenarios increase resource saving}

We explain the resource saving effect of combining two scenarios together. Fig. 1(c) shows that four sources 1, 2, 3, and 4 send their data to the same destination $D$. Each traffic demand from four sources $1,2,3$, and 4 is a unit bandwidth traffic demand and sends a unit bandwidth traffic, where each link cost is 1 . The total number of links used in providing conventional $1+1$ protection for four demands from sources to common destination is 16 and the cost of necessary resources is 16. Links $K-D, O-D, R-D$, and $M-O$ are the common links that can be used in providing 2SD for source node pairs $(1,2),(2,4),(4,3),(3,1)$, respectively. When 2SD for source node pairs $(1,2)$, and $(3,4)$ is used, as shown in Fig. 1(d), the total cost of protection is 14. In Fig. 1(e), we can apply TS for protecting demands from nodes 3 and 4 to destination $D$ since there are four disjoint paths for each, while using conventional $1+1$ protection for demands from nodes 1 and 2. Demand from each node is split into three parts and it costs 0.33 for each link to carry the split traffic, and the total cost is 13.28. In Fig. 1(f), the total cost of protection when using 2SD for source node-pairs of nodes 1 and 2, and TS for nodes 3 and 4 is 12.28 . This demonstrates that more network resources can be saved by using TS and 2SD intelligently. 


\section{Proposed scheme}

We consider a network with $N$ nodes and $L$ undirected links. The proposed scheme is described in the following.

- Step 1: Divide the given traffic matrix into $N$ scenarios, where in each scenario a common destination node has demands from $k$ sources $(2 \leq k \leq N-1)$. For each of these $N$ scenarios, initialize $k=N-1$, and perform step 2 .

- Step 2: Select two sources out of $k$ sources at a time according to the largest effective gain first policy, which is explained in section 3.1.

- Step 2.1: For the selected pair compute the total costs of working and protection paths obtained by using the $2 S D$ scenario and the $T S$ scenario. Select the scenario that provides the minimum total cost.

- Step 2.2: Assign routing to the selected two sources (having a common destination) according the selected scenario. Update $k$ as $k=k-2$, which is the remaining number of source nodes.

- Step 2.3: Select the next two sources from the remaining sources, and assign routing according to steps 3.1-3.2.

- Step 2.4: Repeat steps 3.1-3.3 until $k-2$ equals 1 or 0 . When only 1 source is left, $1+1$ protection with $T S$ scenario is applied for that pair.

- Step 3: The scheme stops.

\subsection{Coding gain}

We describe the largest effective gain first policy to select two sources out of $k$ sources, after defining some notations in the following.

Let $\varphi$ be the set of source nodes having a common destination, where $s_{i} \in \varphi$, $i=1,2, \cdots, k$, where $k \geq 2$. A combination of two sources, $s_{i}$ and $s_{j}$, out of $k$ sources, is expressed by $\left(s_{i}, s_{j}\right) \in \Theta_{2},(i<j)$, where $\Theta_{2}$ is a set of $\left(s_{i}, s_{j}\right)$. $\Theta_{2}$ includes $\left(\begin{array}{l}k \\ 2\end{array}\right)$ combinations of two sources, i.e., pairs.

Coding gain, $\mathcal{G}_{C}\left(s_{i}, s_{j}\right)$, indicates how much network resources is saved in $1+1$ protection by using the $2 \mathrm{SD}$ scenario, consisting of $\left(s_{i}, s_{j}\right)$ and a common destination. Let us assume that $\mathcal{C}_{C}\left(s_{i}, s_{j}\right)$ indicates the cost of employing $1+1$ protection with the $2 \mathrm{SD}$ scenario for $\left(s_{i}, s_{j}\right)$, and $\mathcal{C}_{N O_{-} C}\left(s_{i}, s_{j}\right)$ indicates the same cost without the $2 \mathrm{SD}$ scenario. $\mathcal{G}_{C}\left(s_{i}, s_{j}\right)$ is defined by,

$$
\mathcal{G}_{C}\left(s_{i}, s_{j}\right)=\max \left\{\frac{\mathcal{C}_{N O_{-} C}\left(s_{i}, s_{j}\right)-\mathcal{C}_{C}\left(s_{i}, s_{j}\right)}{\mathcal{C}_{N O_{-} C}\left(s_{i}, s_{j}\right)}, 0\right\} .
$$

Equation (1) states that, if the cost with coding is smaller than that without coding, we achieve some positive gain. Otherwise there is no gain and the solution without using the 2SD scenario is adapted.

Let $\rho_{2}\left(s_{i}, s_{j}\right)$, which is called an effective gain for $2 \mathrm{SD}$, be the product of the coding gain and bandwidth demand for $\left(s_{i}, s_{j}\right) . \rho_{2}\left(s_{i}, s_{j}\right)$, is expressed by,

$$
\rho_{2}\left(s_{i}, s_{j}\right)=\mathcal{G}_{C}\left(s_{i}, s_{j}\right) \times \min \left(\omega_{s_{i} d}, \omega_{s_{j}} d\right),
$$

where $\omega_{s_{i} d}$ and $\omega_{s_{j} d}$ are the traffic demands of source nodes $s_{i}$ and $s_{j}$ to common destination node $d$, respectively. 


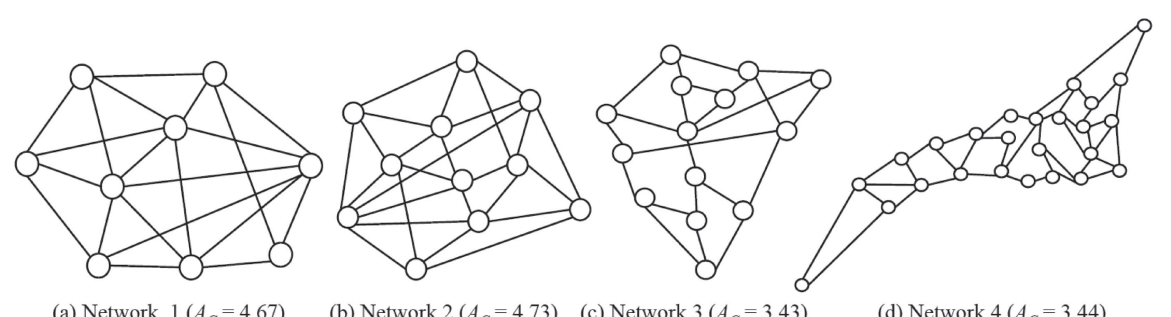

(a) Network $1\left(A_{C}=4.67\right)$

(d) Network $4\left(A_{C}=3.44\right)$

Fig. 2. Examined networks, where $A_{C}$ indicates average node connectivity.

\subsection{Largest effective gain first policy}

- Step 1: For all $\left(s_{i}, s_{j}\right) \in \Theta_{2}$, compute $\rho_{2}\left(s_{i}, s_{j}\right)$.

- Step 2: REPEAT

- Step 2.1: Select $\left(s_{i}, s_{j}\right) \in \Theta_{2}$ with the highest $\rho_{2}\left(s_{i}, s_{j}\right)$.

- Step 2.2: Remove $\left(s_{i}, s_{j}\right)$ and all other pairs including either $s_{i}$ or $s_{j}$ from $\Theta_{2}$.

UNTIL $\Theta_{2}$ is empty.

At the end we select a set of $\left\lfloor\frac{k}{2}\right\rfloor$ pairs with the highest effective gain in each of the case, where $\lfloor x\rfloor$ demotes the largest integer not greater than $x$. The number of times to compute $\rho_{2}\left(s_{i}, s_{j}\right)$ is expressed as $W_{2}(k)$, which is given by,

$$
W_{2}(k)= \begin{cases}\frac{k(k-1)}{2}, & \text { if } k \text { is even } \\ \frac{k(k-1)(k-2)}{2}, & \text { if } k \text { is odd. }\end{cases}
$$

When we select a pair at a time, this policy has the computational complexity of $O\left(k^{2}\right)$ when $k$ is even, and $O\left(k^{3}\right)$ when $k$ is odd.

\section{Results and discussion}

Our evaluation uses networks 1, 2, 3, and 4 in Fig. 2, known as synthetic network [2], COST 239 network [1], Indian network [6], and Japan photonic network (JPN 25) [8], respectively. The traffic demands for all possible source-destination pairs are set equal.

Fig. 3 observes that the proposed scheme achieves more resource saving than each applied individual protection scheme. In networks 1 and 2, the proposed scheme achieves $2.1 \%$ more resource saving than the most efficient scenario

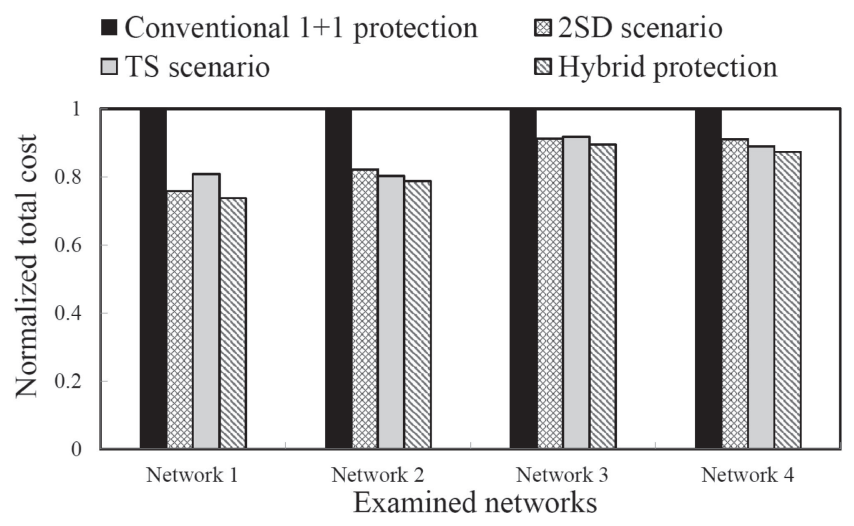

Fig. 3. Comparison of total routing costs. 
between TS and 2SD, and $1.6 \%$ for networks 3 and 4 . The average network connectivity of networks 1 and 2 is higher than that of networks 3 and 4 . In our evaluation, the proposed scheme can saves more resources in the network with higher connectivity. The reason is as follows. 2SD requires that both common destination and transit nodes have the network connectivity with three or more and some specified routes be disjoint [1]. TS requires that both source and destination nodes have the network connectivity with three or more and all the routes be disjoint [2]. Additionally, to make each scenario applicable, its cost with coding must be less than that without coding. The larger the network connectivity is, the higher the possibility for finding more cost-effective routes satisfying the disjoint condition is. When the network connectivity is low, there is less chance that both 2SD and TS are applicable since the above required conditions may not be satisfied. In this case, since our option to take the better scenario out of the two is limited, the resource saving is limited.

We discuss the impacts of computation time and implementation of the proposed scheme, compared to that of individual scenario.

As to the computation time, in the proposed scheme, for each pair both TS and 2SD scenarios are evaluated. Therefore, the computation time of the proposed scheme is at most twice than that of the longer scenario of the two, while the order of the computation time in terms of the numbers of nodes and links are the same as that of individual scenario. As the proposed scheme is used for route design in a planning phase, the double increase of computation time is acceptable for a network designer unless the increase of computation time violates the time constraint in the planning phase. The proposed scheme gives a network designer an option to achieve 1.6-2.1\% resource saving, which leads to reducing the capital expenditure, at the cost of the double increase of computation time.

Second, we consider the implementation impact of the proposed scheme on the encoding and decoding functions. In both $2 \mathrm{SD}$ and TS, each node is required to have encoding and decoding functions. Encoding is performed at intermediate and source nodes in 2SD and TS, respectively. Decoding is performed at a destination node in both $2 \mathrm{SD}$ and TS. In the proposed scheme, each node is also required to have encoding and decoding functions in the same way of both 2SD and TS. Therefore, the implementation complexity of the proposed scheme in terms of encoding and decoding functions is the same as that of each individual scenario.

\section{Summary}

This letter proposed a hybrid scheme to design coding aware $1+1$ protection routes. We observed that the proposed scheme achieves $1.6-2.1 \%$ resource saving than each individual scenario by paying extra computation efforts.

\section{Acknowledgments}

This work was supported in part by the National Institute of Information and Communications Technology, Japan. 